\title{
Nuclear Medicine Technology: Procedures and Quick Reference
}

\author{
P. Shackett \\ Philadelphia, PA: Lippincott, Williams and Wilkins, 2009, 576 pages, \$92.95
}

This is the second edition of a book intended for physicians, residents, nuclear medicine supervisors, technologists, and students interested in developing, modifying, and using imaging and therapy protocols. Each chapter reviews the appropriate radiopharmaceutical and dose, indications and contraindications for the examination, patient preparation, equipment for imaging, protocol, normal and abnormal results, and artifacts. There is also a sample history questionnaire to ensure that the right questions are asked by the physician or technologist when obtaining a patient history.

The book is divided into 2 parts. The first section includes 61 chapters on imaging and therapy protocols that are common to a nuclear medicine department. The second section contains chapters on conversion tables, radiopharmaceuticals and kit preparation, decay tables, drug interventions, laboratory tests, regulations, patient release methods after thyroid therapy, patient history sheets for various examinations, abbreviations commonly used in nuclear medicine, billing codes, and anatomic images. There is also a collection of online images available to the purchaser.

The first section of the book is extremely comprehensive and organized. Every aspect of nuclear medicine imaging protocols is covered and discussed in detail. The second section contains useful tables and information that cover conversion of units, calculation of adult and pediatric doses, Nuclear Regulatory Commission regulations, patient history sheets, and useful information about interventional drugs and their applications. This is one of the most useful books I have found for reviewing protocols and developing in-house patient questionnaires.

The book has been compiled from various imaging protocol sources. As such, there may be some disagreements in imaging times and radiopharmaceutical dosing. However, I feel that this book is an excellent compilation of a standard protocol that would allow an imaging department to expand or delete information from its own imaging protocols. Additionally, the book provides guidelines on developing new protocols.

Although there are several books that cover imaging protocols, such as Nuclear Medicine and PET/CT: Technology and Techniques and Nuclear Medicine: the Requisites, I have encountered no book that is as thorough in its description of how to do a procedure.

COPYRIGHT @ 2012 by the Society of Nuclear Medicine and Molecular Imaging, Inc.

Published online Jun. 18, 2012.

DOI: 10.2967/jnmt.112.104927 